\title{
FENOMENOLOGIA DA DEPRESSÃO: UMA ANÁLISE DA PRODUÇÃO ACADÊMICA BRASILEIRA
}

\author{
Phenomenology of Depression: A Review of Brazilian Academic Production \\ Fenomenología de la Depresión: Una Revisión de la Producción Académica de Brasil
}

Anielli Santiago

Adriano Furtado Holanda

\begin{abstract}
Resumo: O objetivo deste trabalho é apresentar um panorama das pesquisas nacionais sobre depressão na perspectiva fenomenológica. Realizou-se uma pesquisa nas bases de dados virtuais e abertas SciELO (Scientific Eletronic Library Online), PePSIC (Periódicos Eletrônicos em Psicologia) e LILACS (Literatura Latino-Americana e do Caribe em Ciências da Saúde), sendo selecionados e analisados vinte e um artigos, no período de 1981 a 2013. Verificou-se que o assunto é pouco explorado em relação à perspectiva fenomenológica, embora a mesma tenha uma tradição no estudo dos fenômenos psicopatológicos. Além disso, os teóricos clássicos da abordagem são pouco citados nos estudos. Tanto as pesquisas teóricas quanto as empíricas trazem uma diversidade de temas em relação ao transtorno, com destaque para a depressão em idosos. Sugere-se a realização de novas pesquisas e reflexões teóricas que possam dar conta da alta incidência do quadro na população em geral.
\end{abstract}

Palavras-chave: Depressão; Fenomenologia; Produção acadêmica.

Abstract: The objective of this work is to present a panorama of the national researches about depression in phenomenological perspective. We conducted a research on the virtual and opened databases, and at the end of the search, were selected and analyzed twenty-one articles in the period 1981-2013. It was found that the subject is little explored in relation to the phenomenological approach, although it is traditional on the study of psychopathological phenomena. Moreover, classic authors from Phenomenology are rared reported in the studies. Both theoretical and empirical researches bring a diversity of topics in relation to the disorder, especially depression in elderly. We suggest that new researches and theoretical reflections may be realize in order to deal with the high incidence of this problem in general population.

Keywords: Depression; Phenomenology; Academic production.

Resumen: El objetivo del trabajo es presentar una visión general de las encuestas nacionales sobre la depresión en la perspectiva fenomenológica. Se realizó una búsqueda en bases de datos virtuales y abiertas como SciELO (Scientific Electronic Library Online), PePSIC (Revistas Electrónicas en Psicología) y LILACS (Literatura Latinoamericana y del Caribe en Ciencias), se seleccionaron y analizaron veintiún artículos en el período 1981-2013. Se encontró que el sujeto está poco explorado en relación con el punto de vista fenomenológico, a pesar de que tiene una tradición en el estudio de los fenómenos psicopatológicos. Por otra parte, el enfoque clásico teórico rara vez se informó en los estudios. Tanto la investigación teórica como la empírica tocan una diversidad de temas en relación con el trastorno, especialmente la depresión en la tercera edad. Se sugiere llevar a cabo más investigaciones y reflexiones teóricas que pueden explicar la alta incidencia de la trama en la población general.

Palabras-clave: Depresión; la Fenomenología; la Producción académica.

\section{Introdução}

O fenômeno da depressão tem chamado a atenção na atualidade por sua crescente incidência no mundo todo. De acordo com o Relatório sobre a Saúde no Mundo, da Organização Mundial de Saúde (2011), a depressão grave é atualmente a principal causa de incapacitação na população em geral, situando-se em quarto lugar entre as dez principais causas da carga mundial de doenças. De acordo com o mesmo documento, se as projeções se mantiverem corretas, nos próximos vinte anos a depressão deverá ser a segunda das principais causas das doenças no mundo. O Ministério da Saúde calcula que, em um dado mo- mento da vida, entre 13\% e $20 \%$ da população apresenta algum sintoma depressivo; além disso, o custo agregado por prejuízo ao trabalho é imenso (Wannmacher, 2004). Deste fato resulta um número significativo de pesquisas e estudos acerca da temática, de modo a tentar contribuir para o conhecimento de suas causas, formas de tratamento e prevenção (Duarte \& Rego, 2007; Ramires, Passarini, Flores \& Santos, 2009; Vivan \& Argimon, 2009).

O diagnóstico da depressão é complexo, pois leva em conta uma série de sintomas que podem estar associados também a outras patologias. A Classificação Internacional das Doenças (CID-10) faz uma diferenciação entre "episódios depressivos" e "transtorno depressivo recorrente", 
sendo que o que distingue ambos é o tempo e a frequência com que ocorrem. Os sintomas principais das referidas formas de depressão são alteração da capacidade de experimentar o prazer, perda de interesse, diminuição da capacidade de concentração, fadiga acentuada, problemas de sono e diminuição de apetite. Também são frequentes a diminuição da autoestima e autoconfiança e ideias de culpabilidade e/ou indignidade. Além disso, o número e a gravidade de tais sintomas determinam três níveis de episódio e transtorno depressivos: leve, moderado e grave.

O Manual Diagnóstico e Estatístico de Transtornos Mentais (DSM-IV), outro instrumento de referência para diagnósticos, coloca nove critérios para identificar a depressão, sendo eles: estado deprimido a maior parte do tempo, anedonia, sensação de culpa ou inutilidade excessivas, dificuldade de concentração, fadiga, distúrbios do sono, agitação ou lentificação psicomotora, aumento ou redução significativa de peso, e ideias recorrentes de morte e suicídio. De acordo com o número de sintomas, o quadro pode ser classificado em três grupos: "depressão menor" (com a apresentação de dois a quatro sintomas por duas ou mais semanas, incluindo estado deprimido ou anedonia); "distimia” (de três a quatro sintomas, incluindo estado deprimido, durante dois anos, no mínimo) e "depressão maior" (com cinco ou mais sintomas por duas semanas ou mais, incluindo estado deprimido ou anedonia).

Esta complexidade do diagnóstico faz com que as diversas manifestações da depressão muitas vezes se confundam com expressões de depressão não clínicas ou mesmo com estados corriqueiros de tristeza (Parker \& Brotchie, 2009). Mesmo os critérios acima arrolados, constantes no DSM-IV, trazem imprecisões. Como destacam Parker e Brotchie (2009), esse modelo se caracteriza por ser dimensional, onde o estado clínico é definido por parâmetros de gravidade - que é a presença dos sintomas -e requer "a imposição de um ponto de corte que é intrinsecamente impreciso e corre o risco de gerar um diagnóstico de 'falso-positivo' (...) ou avaliação de 'falso-negativo”' (p. 54). Isto torna ainda mais premente a necessidade de não se confundir, ainda, o diagnóstico da depressão com a manifestação de sentimentos de tristeza que acompanham o ser humano em diversos momentos de sua existência.

De acordo com Stefanis e Stefanis (2005), em oposição às respostas emocionais normais a acontecimentos estressantes, a depressão clínica é um transtorno psicopatológico que precisa ser diagnosticado e tratado adequadamente, devido a sua gravidade, recorrência e alto custo para o indivíduo e para a sociedade, além de poder proceder aos encaminhamentos adequados e necessários para seu tratamento. Dessa forma, segundo os autores, é correto utilizar a expressão "transtorno depressivo" para diferenciar a depressão clínica do sentimento normal e transitório de tristeza, bem como utilizar adequadamente a prescrição medicamentosa (Wannmacher, 2004).

É possível verificar, no entanto, que os critérios diagnósticos do DSM-IV para a depressão são baseados no nível de desenvolvimento adulto, porém são utilizados para identificar tal patologia em qualquer idade. Weiss e Garber (2003) defendem o argumento de que se deve levar em conta o grau de desenvolvimento do indivíduo em depressão, visto que " a forma como a depressão é experienciada e expressa depende, em parte, do nível individual de desenvolvimento fisiológico, social e cognitivo" (p. 404). Os autores afirmam que mais estudos na área precisam ser realizados com o objetivo de confirmar a influência das diferenças de desenvolvimento na fenomenologia das depressões, para que se possam produzir novos instrumentos diagnósticos, ou modificar os existentes, no sentido de considerar tais diferenças.

Mari, Jorge e Kohn (2007) apresentam três estudos epidemiológicos que avaliam os índices dos transtornos psiquiátricos em adultos em algumas regiões do Brasil e dos Estados Unidos, sendo estes: (a) uma pesquisa brasileira de morbidade psiquiátrica realizada em Brasília, São Paulo e Porto Alegre, incluindo 6.476 indivíduos com ao menos 15 anos; (b) uma pesquisa que fornece dados sobre os transtornos psiquiátricos em dois bairros da cidade de São Paulo, realizada com 1.462 indivíduos entrevistados; e, (c) o Epidemiological Catchment Area, uma pesquisa em domicílios representativos de cinco regiões dos Estados Unidos, incluindo 17.803 indivíduos com 18 anos ou mais. O índice de prevalência para um ano de depressão maior a partir das três estimativas variou de 3,5 a 9,7\%. O sexo feminino teve os índices mais elevados, variando de 4,7 a $12,6 \%$, enquanto que para o sexo masculino, tais índices variaram de 2,3 a 7\%. Dessa forma, estima-se que o número de pessoas acometidas por depressão maior no ano 2000 esteve entre 5.942 .970 e 15.961.122. Quanto à distimia, o índice de sua prevalência para um ano foi de 1,2\% em São Paulo e 2,8\% nos Estados Unidos. Isso significa que há um número de 2.037 .590 a 4.754 .376 portadores de distimia no Brasil (Mari et al., 2007).

Ballone (2005) aponta como principais fatores de risco para a depressão os seguintes aspectos: histórico familiar de depressão, doença física, episódio anterior de depressão, acontecimentos estressantes ou perdas e abuso de medicamentos ou drogas. Além disso, como apontam Mari et al. (2007), a depressão atinge duas vezes mais mulheres do que homens, em média, sendo que a vulnerabilidade feminina é maior no período pós-parto, tendo em vista que, segundo Higuti e Capocci (2003), cerca de 15\% das mulheres apresentam sintomas depressivos nos primeiros meses que se seguem ao nascimento de um filho.

Retomando o Relatório da Organização Mundial de Saúde (2011), tanto nos países desenvolvidos, onde há uma atenção diferenciada à saúde mental, quanto nos países em desenvolvimento, muitas pessoas que poderiam ser beneficiadas não tiram partido dos serviços psiquiátricos disponíveis. Isso tem bastante relação com o estigma ligado aos indivíduos portadores de transtornos mentais e comportamentais. Várias pesquisas apontam o estigma como um fator que potencializa o sofrimento 
do sujeito em depressão, além de dificultar o diagnóstico e a adesão a um tratamento eficaz (Valentini, Levav, Kohn, Miranda, Mello, Mello \& Ramos, 2004; Moreira, 2007; Moreira \& Melo, 2008). Em um estudo transcultural realizado no Brasil, no Chile e nos Estados Unidos a respeito da experiência do estigma na depressão (Moreira \& Telles, 2008), esse fator aparece relacionado a aspectos como a sensação de não aceitação da depressão, o medo de ser visto como "louco" e a sensação de incapacidade ao ser exigido, o que dificulta bastante o processo de tratamento destas pessoas.

No que concerne ao tratamento da depressão, Souza (1999) aponta que a mesma não pode ser tratada a partir de um modo abstrato, mas a partir do fato de se tratar de pacientes - sujeitos - deprimidos, contextualizados em seus meios sociais e culturais, e compreendidos nas suas dimensões biológicas, psicológicas e sociais. Dessa forma, o autor considera que o tratamento deve ser próprio para cada indivíduo, podendo incluir psicoterapia, mudança de estilo de vida e terapia farmacológica de acordo com a gravidade e características de cada caso. No Brasil, existem os Centros de Atenção Psicossocial (CAPS), que proporcionam atendimento gratuito para pessoas em sofrimento mental cuja severidade as impeçam de realizar suas atividades diárias. Conforme documento do Ministério da Saúde (2004), os CAPS têm como objetivo atender à população em sua área de abrangência, realizando o acompanhamento clínico e a reinserção social dos usuários pelo acesso ao trabalho, lazer, exercício dos direitos civis e fortalecimento dos laços familiares e comunitários. É um serviço de saúde mental criado para substituir as internações em hospitais psiquiátricos.

Pesquisas apontam que a utilização de psicoterapia no tratamento da depressão é amplamente indicada, tanto em casos de intensidade leve e moderada quanto em casos mais graves (Schestatsky \& Fleck, 1999; Berlinck \& Fédida, 2000; Bahls \& Bahls, 2003). Em um estudo de revisão acerca da eficácia da psicoterapia e da farmacoterapia no tratamento da depressão em idosos, Scazufca e Matsuda (2002) encontraram que tratamentos com psicoterapia combinada ou não com medicação foram considerados mais eficazes do que tratamentos farmacoterapêuticos para a depressão maior, mesmo se considerando a necessidade de mais estudos nesta direção. Com isto, vislumbra-se o papel fundamental que a psicologia pode exercer no desenvolvimento de intervenções que possam contribuir para amenizar o sofrimento do paciente em depressão.

A psicologia fenomenológico-existencial é uma abordagem que traz uma compreensão diferenciada dos fenômenos psicopatológicos, em especial quando comparada aos modelos tradicionalmente focados em aspectos biológicos e fisiológicos, centrados numa intervenção nosológica e farmacológica.

O objetivo deste trabalho é discutir e apresentar alguns aspectos da contribuição do enfoque fenomenológico-existencial ao contexto da psiquiatria - em particular
- e da psicologia das depressões, partindo do levantamento e análise das publicações científicas nacionais associadas ao tema. A escolha da temática se justifica pela necessidade de conhecer melhor tal psicopatologia para que se possa tratá-la de modo eficaz, tendo em vista sua rápida expansão na sociedade contemporânea. E a escolha do caminho metodológico se deve à necessidade de reconhecimento do estatuto atual da discussão do tema, no contexto das abordagens fenomenológico-existenciais.

\section{A Tradição Fenomenológica e o Fenômeno da Depressão}

O tema da "depressão" na literatura clássica de psicopatologia e psiquiatria fenomenológicas vem normalmente associado a outros quadros nosológicos. Dentre os primeiros trabalhos de psiquiatras fenomenólogos-existenciais sobre o tema da depressão, podemos citar os nomes de Erwin Straus e Viktor E. Von Gebsattel que, em 1928, publicam - respectivamente - "A Experiência do Tempo na Depressão Endógena e no Desgosto Psicótico" e "Pensamento Pressionado ao Tempo na Melancolia" (Holanda, 2011).

O interesse pelo fenômeno da depressão direciona igualmente as pesquisas de Roland Kuhn, outro psiquiatra da tradição fenomenológico-existencial, para a descoberta dos efeitos antidepressivos da Imipramina em 1954 (Kuhn, 1990/2005). Todavia, os nomes mais conhecidos da tradição fenomenológico-existencial em relação a esta temática são Eugène Minkowski e Ludwig Binswanger.

Minkowski, em 1922, durante uma sessão da Sociedade Suíça de Psiquiatria, em Zurique, apresenta um de seus textos mais famosos: um estudo de caso sobre melancolia esquizofrênica (Minkowski, 1922/1967). Minkowski propõe uma "fenomenologia genético-estrutural”, em que busca esclarecer as conexões e interrelações das vivências patológicas, identificando a estrutura sob a qual se organizam essas vivências. Em seu estudo sobre os pacientes melancólicos, assinala que os sintomas devem ser entendidos a partir da experiência básica do tempo (Cardinali, 2002). Já Binswanger é responsável por um dos textos mais conhecidos sobre esta temática na tradição fenomenológica - o livro Melancolia e Mania. Estudos Fenomenológicos - publicado em 1960.

Contemporaneamente, temos conhecimento dos trabalhos do psiquiatra japonês Kimura Bin que, interessado em Minkowski e Binswanger, passa a estudar os textos de Husserl e Heidegger, chegando a concluir sua formação em psiquiatria na Alemanha (Holanda, 2011). Acerca do tema da depressão, publica um texto intitulado "Fenomenologia da Depressão Estado-Limite" (Bin, 1998). Cabe destacar ainda a figura de Hubertus Tellenbach (1914-1994), que foi um psiquiatra alemão - fez filosofia em Kiel, e medicina e psiquiatria em Munich - que encontrou a Daseinsanalyse de Binswanger, além da antropo- 
logia de Von Gebsattel, Straus e Minkowski. A partir daí, produziu pesquisas sobre melancolia (Holanda, 2011).

Wilhelm Mayer-Gross (1889-1961) foi responsável pelo livro Psiquiatria Clínica, escrito em parceria com E. Slater e M. Ross, que teve sua primeira edição em 1954. Representando o "espírito da escola fenomenológica de Heidelberg”, este livro chega ao Brasil através da tradução de sua terceira edição, de 1969, e “(...) durante muito tempo foi o porto seguro de toda uma geração de psiquiatras, contribuindo para uma virada na direção de uma psiquiatria clínico-fenomenológica na época áurea das correntes antipsiquiátricas das décadas de 1960 e 1970" (Cordas \& Louzã, 2003, p. 116). Mayer-Gross se aproxima desde cedo da fenomenologia, com sua tese defendida em 1913, e intitulada Sobre a fenomenologia dos sentimentos anormais de felicidade (Zur Phänomenologie abnormer Glücksgefühle), tendo sido ainda colega de nomes importantes e representativos do pensamento fenomenológico em psiquiatria, como Karl Jaspers e Hans Gruhle, por exemplo. (Cordas \& Louzã, 2003).

E não podemos esquecer de mencionar a significativa contribuição de Karl Jaspers que, a partir da publicação de sua Psicopatologia Geral, em 1913, praticamente "inaugura" o campo de reflexão conhecido como "psicopatologia fenomenológica” (Jaspers, 1913/1989). Mesmo assim, com tantas contribuições, o campo da "fenomenologia psiquiátrica” ainda é permeado por indefinições (Andreasen, 2007; Mullen, 2007).

No campo da psiquiatria - tradicionalmente - há diferentes usos para a palavra "fenomenologia". Segundo Mullen (2007), “fenomenologia” pode ser tanto a definição precisa dos sintomas psiquiátricos, quanto a descrição das ações e experiências dos pacientes; bem como a busca pelo significado essencial destas experiências ou o exame do "mundo interior" do paciente. Fundamentalmente, compreende-se a "fenomenologia" aplicada à psiquiatria por duas vias (Andreasen, 2007): a) como exame das experiências subjetivas internas e; b) como base para a nosologia psiquiátrica. Em ambos os casos, reconhece-se o lugar ocupado pela proposição de Jaspers de construção de uma "ciência psicopatológica”, tendo como fundamento a Fenomenologia, quando afirma que: "À fenomenologia compete apresentar de maneira viva, analisar em suas relações de parentesco, delimitar, distinguir da forma mais precisa possível e designar com termos fixos os estados psíquicos que os pacientes realmente vivenciam" (Jaspers, 1913/1989, p. 75).

\section{Método}

O presente estudo consistiu em buscar conhecer a produção acadêmica brasileira sobre o tema, no contexto das práticas fenomenológicas e de leituras de orientação fenomenológica, incluindo-se aqui seus desdobramentos na perspectiva existencial. Para tal, foi feita uma revisão bibliográfica realizada a partir de busca de artigos na
Biblioteca Virtual de Saúde - Psicologia (BVS-Psi), juntamente com seus desdobramentos na SciELO (Scientific Eletronic Library Online), PePSIC (Periódicos Eletrônicos em Psicologia) e LILACS (Literatura Latino-Americana e do Caribe em Ciências da Saúde). Inicialmente, foi feita uma pesquisa na BVS-Psi a fim de conhecer o que tem sido produzido sobre a temática da depressão, em geral, no Brasil.

Nesse primeiro momento, foi utilizado apenas o indexador "depressão" para busca de artigos. Foram encontrados 1785 artigos entre as bases de dados SciELO e PePSIC e 6568 artigos na base de dados LILACS. Em um refinamento realizado posteriormente, cruzando o indexador "depressão" com "fenomenologia”, "fenomenológico", "existencial”, "existência” e "fenomenológico-existencial”, foram encontrados 92 artigos nas três bases de dados já citadas. A leitura dos resumos desses artigos permitiu fazer um recorte ainda mais específico: ao final, foram selecionados vinte e um artigos que abordaram a depressão sob uma perspectiva fenomenológica e existencial. Os outros setenta e um artigos foram eliminados pelo fato de serem produções em formato de teses e dissertações e/ou artigos que tratavam de fenômenos envolvidos na depressão (entre eles, fatores neuroquímicos e genéticos, por exemplo), mas que não faziam referência direta ou indireta às perspectivas fenomenológicas e existenciais propriamente ditas.

As vinte e uma publicações selecionadas para a nossa amostra foram submetidas a uma análise qualitativa indutiva e comparativa. A análise foi indutiva por possibilitar a constituição de categorias temáticas derivadas do "contato progressivo com o material" (Pieta, Castro \& Gomes, 2012, p. 132); e comparativa por buscar um diálogo entre os diversos textos. A intenção foi conhecer os conteúdos dessas produções, bem como os caminhos de reflexão - desenvolvidos por pesquisadores brasileiros sobre o tema da "depressão", sob a ótica das perspectivas fenomenológica e existencial.

\section{Resultados e Discussão}

A primeira categorização que pudemos observar divide os artigos em três agrupamentos: a) Estudos Empíricos (dez artigos); b) Estudos Teóricos (dez artigos); e, c) Estudos Epidemiológicos (apenas um artigo). Os estudos empíricos são aqueles que buscam fornecer compreensão, acréscimo ou modificação em determinado tema, utilizando dados coletados a partir de fontes diretas (pessoas) que vivenciam ou têm conhecimento sobre tal tema. Já os estudos teóricos têm como objetivo conhecer ou proporcionar um espaço para discussão de uma temática ou questão, sem utilizar de pesquisa de campo, fundamentalmente a partir de uma reflexão com respeito a aspectos gerais ou específicos de determinada teoria. Os estudos epidemiológicos, por fim, são os que apresentam dados objetivos acerca de alguma patologia, em uma determinada 
região num período de tempo. Para fins de análise, o único texto epidemiológico desta amostra será apresentado juntamente aos textos empíricos.

Não foram encontrados indícios de prevalência de algum periódico sobre o tema, o que pode indicar certa dispersão no que se refere às revistas científicas, já que não há uma grande concentração dos artigos em um ou outro periódico. Apesar disto, observamos uma maior presença de periódicos da área da Saúde - especialmente de Medicina e Enfermagem - como campos propícios ao desenvolvimento do tema. O Jornal Brasileiro de Psiquiatria, a revista Psiquiatria Biológica e a Revista Latinoamericana de Psicopatologia Fundamental/Latin
American Journal of Fundamental Psychopathology On Line tiveram, cada um, três artigos publicados acerca da temática deste estudo, enquanto que a Revista Brasileira de Enfermagem teve dois artigos publicados. Os demais artigos provêm de diversos outros periódicos da psiquiatria e da psicologia, sendo eles: Revista Psico-USF (USF/SP), Revista Mental (Unipac/MG), Revista Aletheia (Ulbra/RS), Revista Brasileira de Epidemiologia (Abrasco), Boletim de Psiquiatria, Estudos e Pesquisas em Psicologia (UERJ), Psicologia: Reflexão e Crítica (UFRGS), Arquivos Brasileiros de Psicologia (UFRJ), Revista Mal Estar e Subjetividade (Unifor/CE) e Saúde e Sociedade (FSP/ USP). A tabela abaixo mostra claramente esta dispersão.

\begin{tabular}{|c|c|c|c|}
\hline Título do artigo & Autor & Revista & Área \\
\hline $\begin{array}{l}\text { Prevalência e fatores associados a sintomas depressivos } \\
\text { em adultos do sul do Brasil: estudo transversal de base } \\
\text { populacional }\end{array}$ & $\begin{array}{l}\text { Rombaldi, Silva, Gazalle, } \\
\text { Azevedo \& Hallal (2010). }\end{array}$ & $\begin{array}{l}\text { Revista Brasileira de } \\
\text { Epidemiologia }\end{array}$ & Epidemiologia \\
\hline $\begin{array}{l}\text { A relação entre variáveis de saúde mental e cognição em } \\
\text { idosos viúvos. }\end{array}$ & $\begin{array}{l}\text { Trentini, Werlang, Xavier \& } \\
\text { Argimon (2009) }\end{array}$ & Psicologia: Reflexão e Crítica & Psicologia \\
\hline $\begin{array}{l}\text { Ressignificação existencial do pretérito e longevidade } \\
\text { humana }\end{array}$ & $\begin{array}{l}\text { Patrício, Hoshino \& Ribeiro } \\
\text { (2009) }\end{array}$ & Saúde e Sociedade & $\begin{array}{l}\text { Diversas áreas } \\
\text { da saúde }\end{array}$ \\
\hline $\begin{array}{l}\text { Os ajustamentos criativos da criança em sofrimento: uma } \\
\text { compreensão da gestalt-terapia sobre as principais psico- } \\
\text { patologias da infância. }\end{array}$ & Antony (2009) & $\begin{array}{l}\text { Estudos e Pesquisas em } \\
\text { Psicologia }\end{array}$ & Psicologia \\
\hline $\begin{array}{l}\text { A contribuição de Tellenbach e Tatossian para uma com- } \\
\text { preensão fenomenológica da depressão }\end{array}$ & Leite \& Moreira (2009) & $\begin{array}{l}\text { Arquivos Brasileiros de } \\
\text { Psicologia }\end{array}$ & Psicologia \\
\hline $\begin{array}{l}\text { Fenomenologia da queixa depressiva em adolescentes: um } \\
\text { estudo crítico-cultural }\end{array}$ & Melo \& Moreira (2008) & Aletheia & Psicologia \\
\hline $\begin{array}{l}\text { Rompimento amoroso, depressão e auto-estima: estudo } \\
\text { de caso. }\end{array}$ & $\begin{array}{l}\text { Guedes, Monteiro-Leitner \& } \\
\text { Machado (2008) }\end{array}$ & $\begin{array}{l}\text { Revista Mal Estar e } \\
\text { Subjetividade }\end{array}$ & Psicologia \\
\hline $\begin{array}{l}\text { Experiências do estigma na depressão: um estudo trans- } \\
\text { cultural }\end{array}$ & Moreira \& Telles (2008) & Psico-USF & Psicologia \\
\hline $\begin{array}{l}\text { Fenomenologia crítica da depressão no Brasil, Chile e } \\
\text { Estados Unidos }\end{array}$ & Moreira (2007) & $\begin{array}{l}\text { Revista Latinoamericana de } \\
\text { Psicopatologia Fundamental }\end{array}$ & Psicologia \\
\hline $\begin{array}{l}\text { Tempo, idade e cultura: uma contribuição à psicopatologia } \\
\text { da depressão no idoso. Parte II: uma investigação sobre a } \\
\text { temporalidade e a medicina }\end{array}$ & Bastos (2006) & $\begin{array}{l}\text { Revista Latinoamericana de } \\
\text { Psicopatologia Fundamental }\end{array}$ & Psicologia \\
\hline Fenomenologia da solidão na depressão & Moreira \& Callou (2006) & Mental & Psicologia \\
\hline $\begin{array}{l}\text { Tempo, idade e cultura: uma contribuição à psicopatologia } \\
\text { da depressão no idoso. Parte I: temporalidade e cultura. }\end{array}$ & Bastos (2005) & $\begin{array}{l}\text { Revista Latinoamericana de } \\
\text { Psicopatologia Fundamental }\end{array}$ & Psicologia \\
\hline $\begin{array}{l}\text { Fenomenologia da criança deprimida: questões existenciais } \\
\text { e biológicas }\end{array}$ & Iserhard (2002) & Psiquiatria biológica & Psiquiatria \\
\hline A descoberta da imipramina e a psicoterapia: uma (re)visão & Souza (1997) & Psiquiatria biológica & Psiquiatria \\
\hline $\begin{array}{l}\text { A depressão nas pessoas idosas: um estudo nosológico } \\
\text { retrospectivo e atual }\end{array}$ & Corrêa (1996) & $\begin{array}{l}\text { Jornal Brasileiro de } \\
\text { Psiquiatria }\end{array}$ & Psiquiatria \\
\hline $\begin{array}{l}\text { Acompanhar um filho hospitalizado: compreendendo a } \\
\text { vivência da mãe }\end{array}$ & Bezerra \& Fraga (1996) & $\begin{array}{l}\text { Revista Brasileira de } \\
\text { Enfermagem }\end{array}$ & Enfermagem \\
\hline $\begin{array}{l}\text { A fenomenologia das depressões: da nosologia psiquiátrica } \\
\text { clássica aos conceitos atuais }\end{array}$ & Corrêa (1995) & Revista Psiquiatria Biológica & Psiquiatria \\
\hline $\begin{array}{l}\text { Um encontro com Heidelberg: personalidade pré-mórbida } \\
\text { e interação conjugal observada de pacientes deprimidos } \\
\text { endógenos }\end{array}$ & $\begin{array}{l}\text { Mundt, Fiedler, Ernest \& } \\
\text { Kohlhof (1994) }\end{array}$ & $\begin{array}{l}\text { Jornal Brasileiro de } \\
\text { Psiquiatria }\end{array}$ & Psiquiatria \\
\hline Classificacao e diagnostico fenomenológico das depressões. & Sá Júnior (1983) & $\begin{array}{l}\text { Jornal Brasileiro de } \\
\text { Psiquiatria }\end{array}$ & Psiquiatria \\
\hline $\begin{array}{l}\text { Sintomas existenciais versus sintomas patológicos, um pro- } \\
\text { blema de rotulagem psiquiátrica: inquérito sobre a vivência } \\
\text { de profissionais da saúde mental }\end{array}$ & Vietta \& Bueno (1987) & $\begin{array}{l}\text { Revista Brasileira de } \\
\text { Enfermagem }\end{array}$ & Enfermagem \\
\hline Abordagem fenomenológico-existencial dos sonhos & Spanoudis (1981) & Boletim de psiquiatria & Psiquiatria \\
\hline
\end{tabular}


Há um aumento significativo no número de publicações a partir dos anos 2000, conforme mostra o gráfico abaixo:

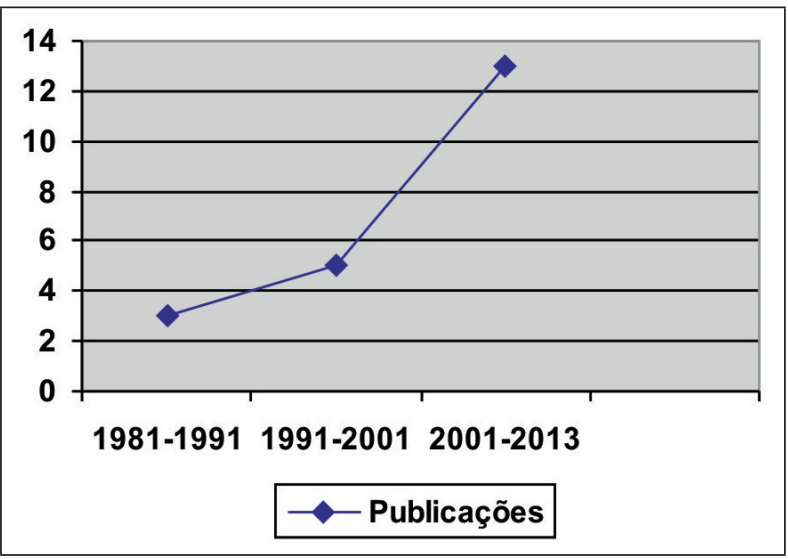

Do total de artigos que compõe a amostra, seis não puderam ser acessados integralmente. Dessa forma, a análise de tais textos foi realizada apenas com base em seus resumos. Três destes artigos foram publicados no Jornal Brasileiro de Psiquiatria, sendo eles: "Classificação e diagnóstico fenomenológico das depressões”, de Sá Júnior (1983); "Um encontro com Heidelberg: personalidade pré-mórbida e interação conjugal observada de pacientes deprimidos endógenos”, de Mundt, Fiedler, Ernest \& Kohlhof (1994) e "A depressão nas pessoas idosas: um estudo nosológico retrospectivo e atual”, publicado por Corrêa (1996). Também não foram encontrados os textos "Fenomenologia da criança deprimida: questões existenciais e biológicas" (Iserhard, 2002); "Abordagem fenomenológico-existencial dos sonhos" (Spanoudis, 1981) e "Acompanhar um filho hospitalizado: compreendendo a vivência da mãe" (Bezerra e Fraga, 1996).

Desses textos que não foram acessados na íntegra, convém destacar que no mais recente deles, Iserhard (2002) trabalha com as perspectivas de Tellenbach e Jaspers. Os demais artigos foram publicados entre os anos 80 e 90 . Spanoudis (1981) faz uma análise da atividade onírica, à luz da "fenomenologia existencial" de Heidegger, a partir de uma paciente depressiva, além de outro paciente psicótico. Sá Júnior (1983) traz uma revisão da classificação e diagnóstico das depressões, na direção de uma "fenomenologia” das síndromes depressivas, numa perspectiva muito próxima da segunda via descrita anteriormente por Andreasen (2007). Correa (1996) caminha na mesma direção de Sá Júnior (1983), mas direcionando a temática para a depressão em idosos, ressaltando a indiferenciação desse quadro em relação aos adultos. Mundt et al. (1994) trazem à discussão o conceito de "tipo melancólico" da escola fenomenológica de Heidelberg - caracterizado por excesso de orientação para a norma e a ordem - num diálogo com a testagem empírica, concluindo pela não homogeneidade do "tipo melancólico", sugerindo inconsistência de avaliações. Finalmente, Bezerra \& Fraga
(1996) procuram realizar uma aplicação do método fenomenológico para a pesquisa das vivências maternas em relação à hospitalização de seus filhos.

É possível notar que também não há uma concentração dos artigos em um autor específico. Apenas três pesquisadores escreveram mais de um artigo da amostra aqui considerada. Isto sugere que no Brasil existem poucos grupos de pesquisa ou mesmo pesquisadores independentes que estudam especificamente o fenômeno da depressão em suas diversas expressões - como é o caso do grupo APHETO da Universidade de Fortaleza (Unifor), que tem dentre seus objetivos desenvolver atividades de pesquisa e extensão em psicoterapia e psicopatologia, bem como em outros campos da clínica, utilizando uma abordagem denominada "humanista-fenomenológica crítica”, utilizando-se de referenciais filosóficos em Merleau-Ponty e Sartre, e psicológicos da Gestalt-Terapia e da Abordagem Centrada na Pessoa ${ }^{1}$.

A seguir, procederemos à análise indutiva das duas macrocategorias: estudos empíricos e estudos teóricos.

\subsection{Artigos Empíricos}

Moreira (2007) desenvolveu um estudo transcultural com o objetivo de compreender a experiência vivida da depressão no Brasil, no Chile e nos Estados Unidos, de modo a constatar possíveis variações culturais do fenômeno. Os resultados mostraram que embora não houvesse grandes variações sintomatológicas entre os três países, a experiência associada a tais sintomas varia conforme traços subjetivos específicos de cada cultura. Deste estudo maior derivaram dois outros: um relativo ao estigma relacionado à depressão nos três países (Moreira \& Telles, 2008) e outro referente ao sentimento de solidão que acompanha a depressão (Moreira \& Callou, 2006), buscando compreender até que ponto a solidão é causa ou consequência de tal psicopatologia. Nestes três estudos, foi utilizado o método fenomenológico crítico para análise das entrevistas, entendendo que tal método tem como objetivo maior compreender a experiência vivida dos indivíduos pesquisados.

Outro artigo publicado pela mesma pesquisadora dos estudos já citados refere-se ao transtorno depressivo em adolescentes (Melo \& Moreira, 2008). O objetivo aqui foi o de compreender a queixa depressiva de vinte adolescentes atendidos pela clínica-escola da Universidade de Fortaleza. As autoras constataram que aspectos culturais, sociais, econômicos e familiares estão fortemente associados à experiência da depressão dos adolescentes entrevistados. Um achado interessante desta pesquisa consiste no fato de que a descrição do que compõe a queixa depressiva diz respeito à forma como a pessoa lida com a experiência da adolescência. Este dado enfatiza a im-

\footnotetext{
Disponível em http://www.apheto.com.br/
}

列 
portância da utilização de um método que procure compreender os fenômenos psicopatológicos para além do diagnóstico clínico, buscando o sentido da experiência descrita pelos indivíduos, tal como o faz o método fenomenológico crítico.

Cinco estudos da amostra aqui considerada tratam da depressão em idosos, sendo dois empíricos e três teóricos. Trentini, Werlang, Xavier \& Argimon (2009) realizaram uma pesquisa com 30 idosos viúvos da cidade de Veranópolis (RS) com o objetivo de avaliar as habilidades cognitivas dos mesmos. Foi utilizado também um grupo controle, constituído por 30 idosos casados. Os autores constataram diferenças significativas entre os idosos viúvos e os casados: o grupo de enlutados diferia dos controles quanto à intensidade de sintomas depressivos e quanto ao escore no Questionário Fenomenológico do Luto. Entre os viúvos, o número médio de sintomas depressivos e a média da intensidade do luto foram significativamente maiores do que os escores observados no grupo controle.

Patrício, Hoshino \& Ribeiro (2009) buscaram determinar os aspectos ambientais envolvidos na longevidade a partir de relatos de ex-ferroviários longevos. Para tanto, os autores utilizaram uma técnica de metodologia qualitativa denominada grounded theory, ou teoria fundamentada nos dados, que é um método que busca as categorias que emergem da fala dos indivíduos. Posteriormente, aglutinam-se tais categorias em outras mais abrangentes, até se chegar às categorias centrais que permitam construir um modelo teórico que dê coerência de significados a todos os dados coletados. Os autores encontraram que as representações dos ex-ferroviários convergem para a categoria central expressa como "Da Vida ao Aniquilamento: o controle social e do estado em defesa da vida". Isso porque a desolação dos indivíduos pesquisados se dá pelo aniquilamento da vida e do ambiente, no presente, causado pela negligência do Estado e da sociedade na promoção e preservação de recursos que existiam no passado. Um resultado interessante encontrado refere-se à hipervalorização do passado, já que além de os fatores geradores de vida terem sido colocados no passado pelos entrevistados, estes reconhecem que participaram da construção de um grande feito sócio-econômico do interior paulista. Assim, a ressignificação do passado, feita pela maioria dos entrevistados, parece ser um dos fatores significativos da longevidade dos mesmos. Os autores asseveram, dessa forma, a importância das correntes psicoterápicas cuja ação central se baseia na construção do significado existencial. Para os idosos entrevistados, tal significado já foi encontrado, sendo necessário haver uma ressignificação. Esta pode ser, muitas vezes, "a única necessidade terapêutica ou ser uma etapa imprescindível do processo terapêutico para construir um novo significado existencial" (Patrício, Hoshino \& Ribeiro, 2009, p. 281).

Guedes, Monteiro-Leitner \& Machado (2008) realizaram um estudo de caso com um paciente acometido por depressão em decorrência do rompimento de um relacionamento amoroso. A abordagem teórica utilizada para fundamentar o estudo foi a centrada na pessoa, mas também lança mão do método fenomenológico para dar suporte à investigação clínica. Fez-se uso de metodologia quantitativa - através de instrumentos padronizados para avaliar as condições psicológicas do cliente - e qualitativa, para captar as "versões de sentido" na fala do mesmo. Como recurso metodológico da prática clínica, a versão de sentido consiste em anotações realizadas pelos psicoterapeutas referindo-se ao momento da sessão, não somente como um registro, mas como relato do vivenciado, impregnado de percepções e impressões sobre a experiência vivida da relação terapêutica. É um instrumento de cunho fenomenológico que propicia a verificação do processo e a revivência da experiência, de modo a proporcionar uma reelaboração dos sentidos presentes na relação subjetiva. Como metodologia, foi descrita por Amatuzzi (1991, 1993, 1995, 1996) para sua aplicação na pesquisa e na formação de profissionais, tendo sido muito utilizada para avaliar efeitos na clínica e na supervisão (Vercelli, 2006; Boris, 2008). De acordo com Guedes et al. (2008), os resultados do processo terapêutico foram positivos, tendo em vista que o paciente aprofundou a percepção de sua condição existencial de responsabilidade pessoal e entrou em contato com sua insegurança, até então identificada com causas externas a si e com os dilemas que vivia, como seus fracassos, dentre eles, nas relações amorosas. Por isso pareceu ressignificar a ideia de "amor" que possuía: eterno, único e imutável. Dessa forma, conseguiu reconhecer e assimilar que para vivenciar uma relação amorosa estável seria preciso se reorganizar e se fortalecer, o que implica, segundo os autores, em conhecer-se mais autenticamente e se lançar no mundo nesta condição mundana.

Outro estudo de caso considerado na amostra se refere ao acompanhamento dos sentimentos, medos e ansiedades vivenciados por uma mãe em situação de hospitalização de seu filho (Bezerra \& Fraga, 1996). A pesquisa indica o uso de alguns elementos da abordagem fenomenológica para análise das entrevistas, mas não especificados no resumo do texto. As autoras constataram que a dedicação e a assistência extremas para com o filho doente deixam a mãe temporariamente descuidada de si mesma. Nesse sentido, a mãe passa a conviver com evidentes sinais de sofrimento psíquico, concretizados em insônia, anorexia e sintomas de depressão. Além disso, a hospitalização da criança é verbalizada pela mãe como algo que tem efeitos sobre toda a família, gerando alterações em sua dinâmica.

Mundt et al. (1994) apresentam as contribuições da escola fenomenológica de Heidelberg, mais especificamente ao trabalho de Willy Mayer-Gros (1889-1961), para a compreensão da depressão. Uma destas contribuições é a delimitação do conceito de "tipo melancólico", que se caracteriza por um excesso de orientação à norma e à or- 
dem, e por aspiração a uma harmonia interior. Existe um instrumento psicométrico, Structural Analysis of Social Behavior (SASB), que permite testar empiricamente tal conceito. O objetivo desta pesquisa foi, portanto, verificar características mais específicas do tipo melancólico em 26 pacientes com diagnóstico de depressão. Amostras de 15 minutos de diálogo entre os pacientes e seus cônjuges forneceram dados interacionais diretamente observáveis que foram, na sequência, analisados pela SASB. Os resultados mostraram que, de fato, pacientes do tipo melancólico procuram harmonia em seus relacionamentos ao evitarem interações crítico-negativas. No entanto, dados de personalidade do Maudsley Personality Inventory (MPI) sugerem que o tipo melancólico não é homogêneo, o que pode levar a dados inconsistentes. Os autores chamam atenção, dessa forma, para a necessidade de realização de novos estudos para quantificar a extensão em que outros aspectos de personalidade se misturam com o tipo melancólico, como bipolaridade ou narcisismo, por exemplo.

Um estudo realizado por Vietta e Bueno (1987) teve como objetivo investigar como os conceitos de normalidade e anormalidade interferem na atuação de docentes de enfermagem. Para tanto, foi necessário investigar quais sintomas são considerados como normais e anormais pela população estudada, bem como a maneira como a mesma se percebe dentro destes referenciais. A metodologia utilizada baseou-se na aplicação de um questionário constando de dados de identificação e questões relativas ao tema proposto, elaboradas a partir de uma listagem de sintomas. Sobre a definição dos conceitos, "considera-se cada um dos sintomas listados no instrumento como situados numa escala representada por um continuum delimitados pelos extremos "normalidade" - "anormalidade" e cuja localização do sintoma, nesta escala, dependerá da intensidade, frequência, situação e momento em que está sendo vivenciado" (Vietta \& Bueno, 1987, p. 258). As autoras encontraram que a população estudada considerou os seguintes sintomas como normais: ansiedade, desânimo, depressão, agressividade, angústia, sentimento de culpa, inferioridade e vontade de agredir. Os sintomas anormais apontados foram delírio, alucinação, ideias obsessivas e vontade de matar. Apareceram como sintomas vivenciados com maior intensidade: depressão, sentimento de inferioridade, perseguição e desânimo. Cabe ressaltar que os sintomas considerados como normais foram aqueles já vivenciados pelos docentes, enquanto que os sintomas ditos anormais foram os nunca vivenciados pelos mesmos. É importante destacar também que alguns sintomas avaliados como normais fazem parte de quadros psiquiátricos pela classificação internacional, como é o caso da ansiedade, por exemplo. $50 \%$ dos indivíduos pesquisados relata ter vivenciado ansiedade em grau intenso e ainda assim isso foi considerado normal. Além disso, as autoras apontam que alguns dos sintomas ditos anormais podem não ter sido admitidos como vivenciados, por se- rem pouco aceitos na sociedade contemporânea. Outro dado interessante é que metade da população se considera equilibrada e a maioria não faz tratamento psicoterápico, mas pensa em se submeter à psicoterapia.

O único estudo epidemiológico desta amostra, realizado por Rombaldi, Silva, Gazalle, Azevedo e Hallal (2010) teve como objetivo identificar a prevalência de sintomas depressivos e fatores associados em uma população de 972 indivíduos na cidade de Pelotas (RS). A análise multivariável indicou que pessoas do sexo feminino, com idade mais avançada, pertencentes a classes sociais menos abastadas, fumantes atuais e que não trabalham, estão, em geral, em maior risco de apresentarem os sintomas em estudo. Os autores ressaltam que conhecer como a sintomatologia depressiva e fatores associados se distribuem na população pode contribuir para o entendimento da fenomenologia dos transtornos depressivos e a traçar estratégias de prevenção e minimização, sendo aqui entendida a "fenomenologia" como metodologia descritiva aplicada aos transtornos mentais.

\subsection{Estudos Teóricos}

Dois estudos teóricos tratam especificamente da depressão na infância. Iserhard (2002) aponta que nos instrumentos diagnósticos usuais não há nenhuma referência à depressão infantil como entidade nosológica própria, na mesma direção do que apontam Weiss e Garber (2003). O autor toma os estudos de Hubertus Tellenbach sobre a situação pré-depressiva e a condição biológica (endon) como base para esclarecer como essa estrutura se manifesta na existência da criança. Além disso, neste estudo faz-se o delineamento do quadro clínico-fenomenológico conforme a concepção psicopatológica de Jaspers e apontam-se formas de tratamento.

Já Antony (2009) apresenta uma compreensão da Gestalt-terapia acerca das principais psicopatologias na infância, a saber, depressão, fobia, transtorno de ansiedade e transtorno obsessivo-compulsivo. Na abordagem gestáltica, segundo a autora, prioriza-se a relação entre homem e mundo; desta forma, os fenômenos psicopatológicos são considerados como oriundos de distúrbios nas relações. Nesta perspectiva, "doença significa perturbações da auto-regulação originadas por mecanismos psicológicos defensivos de contato, que visam inibir a consciência de sentimentos, pensamentos, necessidades, comportamentos que geram angústia e colocam em risco a relação com o outro significativo" (p. 356). O texto ainda aponta que, de maneira geral, as figuras parentais das crianças deprimidas são insuficientes ou inacessíveis para suprir suas necessidades afetivo-emocionais; dessa forma, o trabalho do terapeuta deve caminhar na direção da compreensão fenomenológica da unidade da experiência de todos os envolvidos no contexto em que a criança vive, para que possam tomar consciência de 
suas ações e pensar em formas de ajustamento-criativo frente à patologia.

Em outro estudo teórico, Corrêa (1996) retoma a questão da incidência da depressão em indivíduos idosos, apontando para o fato de que a depressão nessas pessoas apresenta algumas particularidades, porém não difere, em essência, da depressão em adultos. Nesse texto - um dos quais cujo acesso foi limitado ao resumo - a fenomenologia surge como uma tradição na psiquiatria, não se especificando o contexto de sua aplicação.

Bastos (2005; 2006), em dois estudos complementares, buscou relacionar a temporalidade cíclica e a contínua, que são dois aspectos fundamentais das instituições culturais sobre a passagem do tempo, com a psicopatologia, adotando uma visão crítica do construto de depressão no idoso. $\mathrm{O}$ autor baseia seu estudo na experiência clínica e na atitude fenomenológica que orienta essa prática. Nas concepções culturais que percebem a passagem do tempo de maneira predominantemente cíclica, o envelhecer faz parte de um movimento eterno em que a família se perpetua em seus descendentes, em suas tradições, no vínculo com a terra ou no exercício do ofício familiar. As entidades culturais que consideram enfoques mais direcionais da passagem do tempo destacam cada vez mais o papel individual na história social. Quanto mais difícil for a passagem de tendências tradicionais - de caráter circular, fatalista, repetitiva e eterna - para outras de tendência individualizante, burocratizante, planejadora e sucessiva, maiores as dificuldades para um envelhecimento satisfatório e maior a tendência à depressão e medicalização desse fracasso.

Dois artigos discorrem a respeito da questão da classificação e do diagnóstico do transtorno depressivo. Corrêa (1995) apresenta um breve histórico da nosologia das depressões e expõe os conceitos fenomenológicos clássicos das escolas alemã, francesa, suíça e espanhola. O autor explica que as escolas psiquiátricas européias, dentro da tradição kraepeliniana (que considera os fatores ambientais e biológicos como participantes no desenvolvimento da depressão), e baseada na fenomenologia alemã de Jaspers, Kretschmer e Schneider, dividem as depressões em quatro grupos: depressão endógena, determinada por influências hereditárias; depressão situacional, causada por uma situação ambiental perturbadora; depressão neurótica, ocasionada por um conflito emocional interno; e depressão sintomática, causada por doenças corporais e uso de medicamentos ou drogas. Neste estudo, Corrêa (1995) aponta para a necessidade que a psiquiatria do pós-guerra, principalmente em países anglo-saxões, teve de homogeneizar os conceitos dos diversos quadros psiquiátricos de modo a aprimorar os diagnósticos e torná-los mais confiáveis para a realização de um melhor tratamento e de pesquisas e levantamentos epidemiológicos com menor margem de erro. Por fim, as classificações de referência em todo o mundo são abordadas: a classificação americana (DSM-III-R e DSM-IV) e a da Organização Mundial de Saúde (CID-10), que, cada vez mais, caminham para uma aproximação conceitual e descritiva. A nosografia apontada nestes manuais divide o transtorno em depressão maior, distimia, ciclotimia, depressão melancólica, depressão atípica, depressão sazonal e depressão psicótica.

Sá Júnior (1983) faz uma revisão sobre a classificação e o diagnóstico da depressão partindo das ambiguidades desse conceito na atualidade e chegando à diferenciação entre a depressão enquanto sintoma, a depressão enquanto doença e a síndrome depressiva. As classificações clínicas das síndromes depressivas, desde as nosológicas, as propostas pela CID e as que consideram a intensidade do quadro clínico são revistas ao lado de uma classificação fenomenológica de tais síndromes. Com base nesses estudos, o autor propõe diretrizes para diagnosticar as depressões, avaliar os sintomas, verificar as formas de evolução, a presença de queixas somáticas e a intensidade da síndrome e avaliar os fatores biológicos, psicológicos e sociais que fazem parte da síndrome depressiva.

Levando em conta uma visão fenomenológica em psicopatologia, que propõe, sobretudo, o contato com a experiência vivida do indivíduo em sofrimento mental, Leite \& Moreira (2009) apresentam a contribuição de Hubertus Tellenbach e de Arthur Tatossian para a compreensão do transtorno depressivo (como igualmente descrito em Iserhard, 2002). Tellenbach - segundo as autoras - considera o caráter endógeno dos fenômenos psicopatológicos a partir do conceito de endon, que é o terceiro campo etiológico do humano, ao lado do somático e do psíquico. Nessa concepção, endon se refere à corporeidade humana enquanto global, isto é, aquilo que tem caráter vital para o indivíduo e se apresenta como unidade. Além disso, Tellenbach fala a respeito do typus melancholicus como característico do indivíduo depressivo (Leite \& Moreira, 2009). Esse tipo está relacionado a uma rigidez, a um estar fixado, o que, muitas vezes, está relacionado à vida profissional dessas pessoas: 0 trabalho é uma tarefa a ser cumprida com a maior perfeição. Para Tellenbach, a ordenalidade em que o tipo melancólico vive imerso apresenta caracterização patológica, já que predispõe a imposição de limites rígidos, que dificilmente são transcendidos. É dessa forma que se apresentam, em grande parte, as pessoas em depressão: presas em limites autoimpostos e restritas em sua corporalidade (Leite \& Moreira, 2009).

Segundo as autoras, Tatossian concebe o indivíduo depressivo como um ser marcado por uma impossibilidade de se fazer presente em sua própria existência. Para este autor, o depressivo assiste à sua tristeza, sendo incapaz de entrar em relação com ela. Tal incapacidade se estende a toda ação, o que ocasiona uma inibição vital e um vazio temporal. É como se nem mesmo a tristeza pudesse ser sentida pelo indivíduo, que não experimenta mais do que um sentimento de vazio. Além disso, Tatossian afirma que no estado depressivo há uma alteração do tempo 
vivido. Assim, o não fazer e o não ser têm relação direta com a estagnação do tempo vivido, já que na experiência da depressão a existência é lançada no vazio (Leite \& Moreira, 2009).

Souza (1997) revisa duas conferências proferidas por Roland Kuhn, uma em 1972 (realizada em Belo Horizonte) e outra em 1977 (em Barcelona). Evidencia-se, nestas conferências, como a descoberta da imipramina esteve estritamente relacionada à prática da psicoterapia. Kuhn foi o responsável pela descoberta dos efeitos antidepressivos da imipramina, sendo esta descoberta respaldada no conceito de “depressão vital” de Ludwig Binswanger. Este conceito refere-se a um distúrbio funcional do organismo, apresentando uma sintomatologia que perturba funções vitais. Conforme Kuhn, apoiando-se em Binswanger, os distúrbios vitais do humor, entre eles a depressão vital, deveriam ser submetidos a uma terapia farmacológica, principalmente quando houver histórico familiar de algum destes transtornos. Para o tratamento de alguns casos de depressão vital, a psicoterapia se limitaria a dar apoio à farmacoterapia, apenas atuando como fonte de informações sobre os medicamentos e resolução de situações proporcionadas pelo distúrbio. Em outros casos, a psicoterapia combinada com o medicamento se mostrou essencial. Para a análise existencial, Kuhn assinalou que o importante é conhecer os fatores que determinam o curso da história de vida. Corrêa (1995) traz, por fim, a discussão de Kuhn sobre a possibilidade de o próprio terapeuta prescrever o medicamento, apoiado nos argumentos de que o terapeuta possui uma maior influência sobre o paciente e está em melhores condições de observá-lo em sua interação com a medicação, além de a medicação tornar a psicoterapia mais fácil, rápida e satisfatória para o paciente e para o terapeuta.

Por fim, temos um texto de Spanoudis (1981), no qual se apresenta uma compreensão fenomenológico-existencial acerca dos sonhos, a partir de alguns exemplos de uma paciente depressiva e de um paciente psicótico.

\section{Considerações Finais}

É possível notar que o número de artigos encontrados no primeiro momento, nas três bases de dados pesquisadas, utilizando apenas o indexador "depressão" para busca, é significativo. No entanto, este número se apresenta muito restrito ao se buscar artigos sobre esta temática em relação a uma abordagem específica, como é a fenomenologia-existencial. Embora esta perspectiva tenha uma tradição no estudo dos fenômenos psicopatológicos, pudemos verificar que a produção acadêmica sobre a depressão no Brasil utilizando esta abordagem é bastante pequena, o que contrasta com os dados alarmantes apresentados a respeito deste quadro.

Um dos possíveis motivos desse número pouco expressivo pode estar relacionado à própria indefinição do campo - como descrito por Andrease (2007) e Mullen (2007) -, o que faz com que uma "fenomenologia" da depressão possa simplesmente ser encarada como nosologia psiquiátrica, conforme apontado anteriormente, e, assim, não apareça claramente nos indexadores. Ademais, esta consideração aproxima sobremaneira uma perspectiva fenomenológico/nosológica da vertente biológica. Há dificuldades no estudo da fenomenologia filosófica tanto na própria filosofia quanto na psicologia e na medicina, além de haver uma pequena tradução dos textos existentes no Brasil.

Esta indefinição pode ser constatada por este levantamento, quando encontra desde a vinculação da fenomenologia aos modos de classificação e diagnóstico psicopatológicos - como temos em Sá Júnior (1983) ou Correa (1995), p. ex. - e, portanto, associados a uma "fenomenologia descritiva"; quanto a sua associação a um modo de acesso ao "vivido" ou à "experiência vivida" - como encontramos em Melo e Moreira (2008) ou Leite e Moreira (2009), p. ex. - aproximando-se de uma "fenomenologia compreensiva”. As vinculações - nem sempre bem delimitadas - da Fenomenologia com o movimento existencial - são também referências dessa indefinição, e igualmente se apresentam em nossa amostra, como em Patrício et al. (2009), por exemplo.

No entanto, mesmo com o reconhecimento que o levantamento é limitado pelo número de vinte e um artigos (o que remete à necessidade de um posterior levantamento mais aprofundado, e que inclua as demais produções acadêmicas, em especial as dissertações e teses de pós-graduações, bem como uma metanálise associada a produções internacionais), podemos ter um vislumbre das pesquisas sobre depressão que adotam a abordagem fenomenológico-existencial, no Brasil.

Em relação aos estudos empíricos, alguns deles abordam a depressão em relação a uma população específica - idosos, adolescentes, docentes (Trentini et al., 2009; Patrício et al., 2009; Melo \& Moreira, 2008; Vietta \& Bueno, 1987) -, enquanto outros tratam de eventos que possam vir a desencadear a depressão ou fatores relacionados a ela - estigma, solidão, rompimento amoroso, internação de um filho (Moreira \& Telles, 2008; Moreira \& Callou, 2006; Guedes et al., 2008; Bezerra \& Fraga, 1996). Grande parte destes estudos empíricos considera, a partir de dados coletados e analisados, que embora não haja variação na sintomatologia do transtorno depressivo, existem algumas diferenças no que se refere às vivências pessoais relacionadas a este quadro que variam de acordo com os processos subjetivos característicos de cada cultura (Moreira, 2007; Moreira \& Telles, 2008; Patrício et al., 2008; Vietta \& Bueno, 1987; Moreira \& Callou, 2006; Melo \& Moreira, 2008).

Os estudos teóricos também se concentram em torno de algumas temáticas peculiares, entre elas, a depressão em crianças (Iserhard, 2002; Antony, 2009), a depressão em idosos (Corrêa, 1996; Bastos, 2005, 2006), a classifi- 
cação e o diagnóstico dos quadros depressivos (Corrêa, 1995; Sá Júnior, 1983) e a contribuição de alguns autores clássicos da fenomenologia para o entendimento da psicopatologia em questão (Leite \& Moreira, 2009; Souza, 1997). Alguns autores pontuam a necessidade de se considerar as particularidades das diferentes fases da vida na avaliação para diagnóstico do transtorno depressivo, tendo em vista que os manuais diagnósticos universalizam os sinais e sintomas relacionados ao quadro (Iserhard, 2002; Corrêa, 1996; Sá Júnior, 1983).

É interessante destacar que, dos vinte e um artigos da amostra, cinco são assinados pela pesquisadora Virgínia Moreira, da Universidade de Fortaleza, o que representa um número considerável. No entanto, a dispersão dos demais autores indica uma não-concentração dos estudos em um ou outro pesquisador. O mesmo vale para os periódicos em que os estudos foram publicados. Percebese que não há centralização em alguma revista científica em particular.

Outro dado que chama a atenção é a igual "dispersão" de teóricos que poderiam apoiar ou referendar o campo de pesquisas sobre depressão. São pouco citados os teóricos "clássicos", como Jaspers (tradicionalmente associado ao modelo descritivo de psicopatologia e psiquiatria), bem como Bisnwanger e Minkowski. Não foram encontradas referência a Von Gebsattel ou a Straus; porém, chama a atenção a menção a Roland Kuhn (particularmente por seus estudos sobre a Imipramina), em dois artigos (Correa, 1995; Souza, 1997); e a presença forte de Hubertus Tellenbach como representante mais contemporâneo da perspectiva fenomenológica em psiquiatria (Mundt et al., 1994; Iserhard, 2002; Leite \& Moreira, 2009), bem como seu discípulo - Arthur Tatossian (Leite \& Moreira, 2009) - o que pode representar tanto uma "redescoberta" da fenomenologia no campo da psicopatologia, como a indicação de um caminho de "retomada" desta mesma fenomenologia na contemporaneidade.

Um dado relevante com relação à amostra é a presença de cinco estudos tratando da depressão em idosos, o que parece indicar o reconhecimento da incidência considerável desse transtorno nesta população específica. De fato, conforme dados de uma pesquisa realizada por Snowdon (2002), cerca de 10\% dos idosos em todo o mundo apresentam quadros depressivos. No Brasil, este número é ainda maior, passando dos 15\%. Esses dados sugerem a importância de se dar uma atenção especial à saúde mental dos indivíduos da terceira idade. Todavia, dada a alta incidência da depressão na população em geral, chama-nos ainda a atenção a pouca produção associada à população infantil e adolescente, bem como na própria população adulta. Esse dado é ainda mais relevante quando se tomam as conclusões de reconhecer que os critérios diagnósticos do DSM-IV para a depressão são baseados no nível de desenvolvimento adulto, mas utilizados para identificar a patologia em qualquer idade (Weiss \& Garber, 2003).
Por fim, espera-se que este estudo possa servir como "retrato" da necessidade de realização de novas pesquisas empíricas - preferencialmente associadas ao campo médico - e de novas reflexões teóricas que possam dar conta da alta incidência dos transtornos do espectro depressivo na população em geral. Tanto as pesquisas empíricas quanto as reflexões teóricas, devem vir acompanhadas de uma clara e ampla descrição contextual (neste caso, novos estudos epidemiológicos, dirigidos para populações específicas são igualmente desejáveis), de modo que seja possível traçar metas razoáveis de atenção e políticas públicas condizentes com as necessidades da população. No que tange especificamente ao domínio dos estudos fenomenológicos, e em consideração ao extenso histórico dessa perspectiva no contexto das práticas psicológicas e psiquiátricas, reconhece-se uma carência igualmente significativa de estudos no país, o que parece apontar para uma limitada penetração do pensamento fenomenológico na Psiquiatria e Psicologia brasileiras.

\section{Referências}

Amatuzzi, M. M. (1991). O Sentido-Que-Faz-Sentido: Uma pesquisa fenomenológica do processo terapêutico. Psicologia, Teoria e Pesquisa (Brasília), 7(1), 1-12.

Amatuzzi, M. M. (1993). Etapas do Processo Terapêutico: Um estudo exploratório. Psicologia, Teoria e Pesquisa (Brasília), 9(1), 1-21.

Amatuzzi, M. M. (1995). Descrevendo processos pessoais. Estudos de Psicologia (Campinas), 12(1), 65-79.

Amatuzzi, M. M. (1996). O Uso da Versão de Sentido na Formação e Pesquisa em Psicologia. Coletâneas da Anpepp (pp. 11-25). Vol. 1, Nr. 9 (Setembro). Campinas: Editora Alínea.

American Psychiatric Association. (1998). DSM-IV: Manual diagnostico e estatístico de transtornos mentais ( $4^{\text {th }}$ ed.). Porto Alegre, RS: ArtMed.

Antony, S. M. R. (2009). Os ajustamentos criativos da criança em sofrimento: uma compreensão da gestalt-terapia sobre as principais psicopatologias da infância. Estudos e Pesquisas em Psicologia, 9(2), 356-375.

Andreasen, N. C. (2007). DSM and the Death of Phenomenology in America: An Example of Unintended Consequences. Schizophrenia Bulletin, 33(1), 108-112.

Bahls, S. C., \& Bahls, F. R. C. (2003). Psicoterapias da depressão na infância e na adolescência. Estudos de Psicologia (Campinas), 20(2), 25-34.

Ballone, G. J. (2005). Causas da depressão. Disponível em http:// www.psiqweb.med.br/ Acesso em 01 de junho de 2012.

Bastos, C. L. (2005). Tempo, idade e cultura: uma contribuição à psicopatologia da depressão no idoso. Parte I: temporalidade e cultura. Revista Latinoamericana de Psicopatologia Fundamental, 8(4), 738-753. 
Bastos, C. L. (2006). Tempo, idade e cultura: uma contribuição à psicopatologia da depressão no idoso. Parte II: uma investigação sobre a temporalidade e a medicina. Revista Latinoamericana de Psicopatologia Fundamental, 9(1), 126-137.

Berlinck, M. T., \& Fédida, P. (2000). A clínica da depressão: questões atuais. Revista Latinoamericana de Psicopatologia Fundamental, 3(2), 09-25.

Bezerra, L. F. R., \& Fraga, M. N. O. (1996). Acompanhar um filho hospitalizado: compreendendo a vivência da mãe. Revista Brasileira de Enfermagem, 49(4), 611-624.

Bin, K. (1998). Fenomenologia da Depressão Estado-Limite, Revista Latinoamericana de Psicopatologia Fundamental, 1(3), 11-32.

Boris, G. D. J. B. (2008). Versões de Sentido: Um instrumento fenomenológico-existencial para a supervisão de psicoterapeutas iniciantes. Psicologia Clínica (Rio de Janeiro), 20(1), 165-180.

Cardinali, I. R. (2002). Daseinsanalyse e Esquizofrenia. São Paulo: EDUC.

Cordas, T. A. \& Louzã, M. R. (2003). Willy Mayer-Gross (18891961). Revista de Psiquiatria Clínica, 30(4), 116-120.

Corrêa, A. C. O. (1995). A fenomenologia das depressões: da nosologia psiquiátrica clássica aos conceitos atuais. Revista Psiquiatria Biológica, 3(3), 61-72.

Corrêa, A. C. O. (1996). A depressão nas pessoas idosas: um estudo nosológico retrospectivo e atual. Jornal Brasileiro de Psiquiatria, 45(6), 335-343.

Duarte, M. B. \& Rego, M. A. V. (2007). Comorbidade entre depressão e doenças clínicas em um ambulatório de geriatria. Cadernos de Saúde Pública (Rio de Janeiro), 23(3), 691-700.

Guedes, D. D.; Monteiro-Leitner, J., \& Machado, K. C. R. (2008). Rompimento amoroso, depressão e auto-estima: estudo de caso. Revista Mal-estar e Subjetividade, 8(3), 603-643.

Higuti, P. C. L., \& Capocci, P. O. (2003). Depressão pós-parto. Revista de Enfermagem da UNISA, 4, 46-50.

Holanda, A. F. (2011). Gênese e Histórico da Psicopatologia Fenomenológica. Em V. Angerami-Camon (Org.), Psicoterapia e Brasilidade [pp. 115-160]. São Paulo: Cortez Editora.

Iserhard, R. F. (2002). Fenomenologia da criança deprimida: questões existenciais e biológicas. Revista Psiquiatria Biológica, 10(4),161-168.

Jaspers, K. (1989). Psicopatologia Geral. Rio de Janeiro: Livraria Atheneu (Original publicado em 1913).

Kuhn, R. (2005). Psicofarmacologia e análise existencial. Revista Latino-Americana de Psicopatologia Fundamental, 8(2), 221-243 (Original publicado em 1990).

Leite, M. E. \& Moreira, V. (2009). A contribuição de Tellenbach e Tatossian para uma compreensão fenomenológica da depressão. Arquivos Brasileiros de Psicologia, 61(3), 46-56.
Mari, J. J.; Jorge, M. R., \& Kohn, R. (2007). Epidemiologia dos transtornos psiquiátricos em adultos. Em M. F. Mello, A. A. F. Mello e R. Kohn (Orgs.), Epidemiologia da saúde mental no Brasil (pp. 119-141). Porto Alegre: Artmed.

Melo, A. K. S. \& Moreira, V. (2008). Fenomenologia da queixa depressiva em adolescentes: um estudo crítico-cultural. Aletheia, 27(1), 51-64.

Ministério da Saúde. (2004). Saúde mental no SUS: os Centros de Atenção Psicossocial. Brasília: Ministério da Saúde.

Minkowski, E. (1967). Hallazgos de un caso de depresión esquizofrénica. Em R. May, E. Angel \& H. Ellenberger (Orgs.), Existéncia (pp. 163-176). Madrid: Editorial Gredos (Original publicado em 1922).

Moreira, V. (2007). Critical phenomenology of depression in Brazil, Chile and the United States. Journal of LatinAmerican Fundamental Psychopathology online, 7(2), 193-218.

Moreira, V. \& Callou, V. (2006). Fenomenologia da solidão na depressão. Mental, 4(7), 67-83.

Moreira, V. \& Melo, A. K. (2008). "Minha doença é invisível": revisitando o estigma de ser doente mental. Interação em Psicologia, 12(2), 307-314.

Moreira, V. \& Telles, T. C. B. (2008). Experiências do estigma na depressão: um estudo transcultural. Psico-USF, 13(2), 233-241.

Mundt, C.; Fiedler, P.; Ernest, S. \& Kohlhof, A. (1994). Um encontro com Heidelberg: personalidade pré-mórbida e interação conjugal observada de pacientes deprimidos endógenos. Jornal Brasileiro de Psiquiatria, 43(6), 303-309.

Mullen, P. E. (2007). A Modest Proposal for Another Phenomenological Approach to Psychopathology. Schizophrenia Bulletin, 33(1), 113-121.

Organização Mundial da Saúde. (1997) CID-10 Classificação Estatística Internacional de Doenças e Problemas Relacionados à Saúde (10 Rev., vol. 1). São Paulo: Universidade de São Paulo.

Organização Mundial de Saúde. (2011). Relatório sobre a saúde no mundo. Genebra: Organização Mundial de Saúde.

Patrício, K. P., Hoshino, K. \& Ribeiro, H. (2009). Ressignificação existencial do pretérito e longevidade humana. Revista Saúde e Sociedade, 18(2), 273-283.

Parker, G. \& Brotchie, H. (2009). Depressão Maior suscita questionamento maior. Revista Brasileira de Psiquiatria, 31 (Supl.I), 53-56.

Pieta, M. A.; Castro, T. G. de \& Gomes, W. B. (2012). Psicoterapia e Pesquisa: Desafios para os Próximos 10 anos no Brasil. Em A. F. Holanda (Org.), O Campo das Psicoterapias. Reflexões Atuais [pp. 121-142]. Curitiba: Juruá Editora.

Ramires, V. R. R., Passarini, D. S., Flores, G. G. \& Santos, L. G. (2009). Fatores de risco e problemas de saúde mental de crianças. Arquivos Brasileiros de Psicologia (Rio de Janeiro), 61(2), 1-14. 
Rombaldi, A. J., Silva, M. C., Gazalle, F. K., Azevedo, M. R., \& Hallal, P. C. (2010). Prevalência e fatores associados a sintomas depressivos em adultos do sul do Brasil: estudo transversal de base populacional. Revista Brasileira de Epidemiologia, 13(4), 620-629.

Sá Júnior, L. S. M. (1983). Classificação e diagnostico fenomenológico das depressões. Jornal Brasileiro de Psiquiatria, 32(6), 351-358.

Scazufca, M., \& Matsuda, C. M. C. B. (2002). Revisão sobre a eficácia de psicoterapia vs. farmacoterapia no tratamento de depressão em idosos. Revista Brasileira de Psiquiatria, 24(1), 64-69.

Schestatsky, S. \& Fleck, M. (1999). Psicoterapia das depressões. Revista Brasileira de Psiquiatria, 21(1), 41-47.

Snowdon, J. (2002). Qual é a prevalência de depressão na terceira idade? Revista Brasileira de Psiquiatria, 24(1), São Paulo.

Souza, C. A. C. (1997). A descoberta da imipramina e a psicoterapia: uma (re)visão. Revista Psiquiatria Biológica, 5(1), 33-8.

Souza, F. G. M. (1999). Tratamento da depressão. Revista Brasileira de Psiquiatria, 21(1), São Paulo.

Spanoudis, S. (1981). Abordagem fenomenológico-existencial dos sonhos. Boletim de Psiquiatria, 14(2), 51-6.

Stefanis, C. N., \& Stefanis, N. C. (2005) Diagnóstico dos transtornos depressivos: uma revisão. Em M. Maj \& N. Sartorius (Orgs.), Transtornos depressivos (pp. 13-76). Porto Alegre: Artes Médicas.

Trentini,, C. M., Werlang, B. S. G., Xavier,, F. M. F., \& Argimon, I. I. L. (2009). A relação entre variáveis de saúde mental e cognição em idosos viúvos. Psicologia: Reflexão e Crítica, 22(2), 236-243.

Valentini, W., Levav, I., Kohn, R., Miranda, C. T., Mello, A. A. F., Mello, M. F., \& Ramos, C. P. (2004). Treinamento de clínicos para o diagnóstico e tratamento da depressão. Revista de Saúde pública, 38(4), 522-528.
Vercelli, L. C. A. (2006). Versões de Sentido: Um instrumento metodológico, Cadernos de Pós-Graduação - Educação (São Paulo), 5(1), 191-195.

Vietta, E. P., \& Bueno, S. M. V. (1987). Sintomas existenciais versus sintomas patológicos, um problema de rotulagem psiquiátrica: inquérito sobre a vivência de profissionais da saúde mental. Revista Brasileira de Enfermagem, 40(4), 256-262.

Vivan, A. S., \& Argimon, I. I. L. (2009). Estratégias de enfrentamento, dificuldades funcionais e fatores associados em idosos institucionalizados. Cadernos de Saúde Pública, 25(2), 436-444.

Wannmacher, L. (2004). Depressão Maior: Da descoberta à Solução? Uso Racional de Medicamentos. Temas Selecionados (Ministério da Saúde), 1(5), pp. 1-6 (Abril). Disponível em http://bvsms.saude.gov.br/bvs/publicacoes/ HSE_URM_DEP_0404.pdf

Weiss, B., \& Garber, J. (2003). Developmental differences in the phenomenology of depression. Cambridge Journals Online - Development and Psychopathology, 15, 403-430.

Anielli Santiago - Graduanda em Psicologia pela Universidade Federal do Paraná. Email: anielli.sant@hotmail.com

Adriano Furtado Holanda - Doutor em Psicologia, Professor Adjunto do Departamento de Psicologia e do Programa de Pós-Graduação em Psicologia da Universidade Federal do Paraná (UFPR). Endereço Institucional: Departamento de Psicologia, Universidade Federal do Paraná. Praça Santos Andrade, 50 - Sala 215 (Ala Alfredo Buffren). 80020.300. Curitiba/PR. Email: aholanda@yahoo.com

Recebido em 19.04.13 Primeira Decisão Editorial em 16.05.13 Aceito em 30.06.13 